purified preparations indicated the presence of acetyl glucosamine. (We are grateful to Mr. A. Bennett for some of the acetyl estimations.) Possibly the carbohydrate component other than glucosamine was galactose or a mixture of glucose and mannose ${ }^{2}$. Our more recent colorimetric analysis ${ }^{10}$ indicated mannose and galactose, which, with acetylglucosamine and fucose, have been isolated from hydrolysates of blood group $A$ substance ${ }^{11}$

It remains to be seen whether $C l$. welchii filtrates will also affect chorionic and pituitary gonadotrophins.

Wellcome Research Laboratories, Beckenham, Kent. May 6.

*Present address: British Chemicals and Biologicals, Ltd., Benger's, Itd., Holmes Chapel, Cheshire.

1 Whitten, W. K., Nature, 163, 534 (1949).

"Oakley, C. I., Warrack, G. H., and Warren, M. E., J. Path. Bact., $60,495(1948)$

${ }^{3}$ Adams, M. H., and Hendee, E. D., J. Immunol., 51, 249 (1945).

4 Bidwell, E., unpublished results (1947).

S Stack, M. V., and Morgan, W. T. J., Biochem. J., 43, Proc. li (1948)

'Bussard, A., and Eyquem, A., Ann. Inst. Pasteur, 73, 1194 (1947).

${ }^{7}$ Fleischer, G., Schwenk, E., and Meyer, K., Nature, 142, 835 (1938).

${ }^{8} \mathrm{Li}, \mathrm{C} . \mathrm{H}$., Evans, H. M., and Wonder, D., J. Gen. Physiol., 23, 733 (1940).

' Gurin, S., and Hood, D. B., J. Biol. Chem., 131, 211 (1939).

${ }^{10}$ Friedmann, R., Biochem. J., 44, 117 (1949).

${ }^{11}$ Bray, H. G., Henry, H., and Stacey, M., Biochem. J., 40, 124 (1946).

\section{Plastic Deformation of Glass during Scratching}

ReCentry a short note by E. W. Taylor on plastic deformation of optical glass ${ }^{1}$ has appeared. In this connexion a photomicrograph of scratches made on an ordinary microscope slide glass by a diamond is shown in Fig. 1. The diamond was shaped in the form of a cube with its faces parallel to cube planes and one cube corner was used as the scratching point, so that a body diagonal of the cube was perpendicular to the glass slide during scratching. In this position a (110) plane of the cube is also perpendicular to the slide, and the direction of scratching lies in this plane. The scratches, as reproduced, were made with an edge of the cube leading (the other possibility being that a face is leading). The angle between the cube edge and the plane of the glass slide was about $35^{\circ}$ (ideal value $35^{\circ} 16^{\prime}$ ).

The most remarkable feature of this micrograph is the appearance of turnings at the ends of the scratches. If it were not known that these scratches are made in glass, it might be supposed that they are formed in an ordinary plastic material. The turnings are curled like metal turnings and they show the same smoothness.

Another remarkable point is the observation that these turnings are only found at definite loads. In this particular test, no turnings were found for loads varying from 1 to $15 \mathrm{gm}$.; they occurred only at 20,25 and $38 \mathrm{gm}$. At the highest load used, the turnings are not nearly so long as at the two lower loads applied, because they easily break into shorter pieces, as can be seen from Fig. 2.

In addition, no turnings were found if a cube face was leading during scratching. It may be that the wedge action of the cube edge, if this is leading, bears some relation to the formation of the turnings.

At the lower loads of $1-15 \mathrm{gm}$., only very fine chips were formed, and they are seen to be deposited

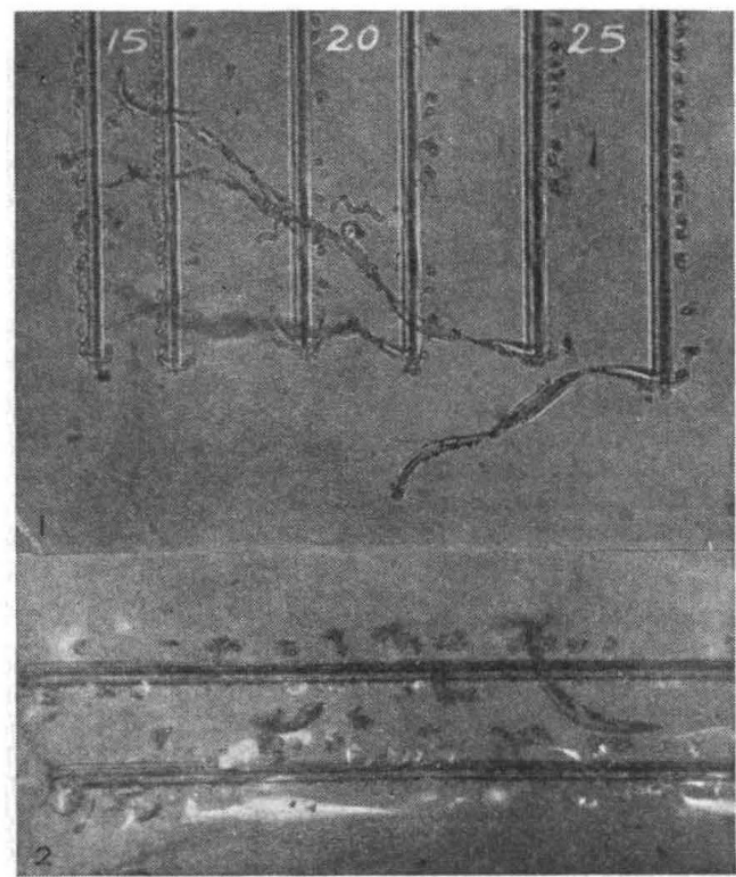

Fig. 1. Seratches made in glass by a diamond point. Magnification: $\times 385$. Actual width of scratch $17 \cdot 5$ microns at load $25 \mathrm{gm}$.

Fig. 2. Scratch made in glass by a diamond point. Magnifleation : $\times 350$. Load $38 \mathrm{gm}$

along the side of the scratch. Such fine chips can also be seen, however, in those cases where turnings are formed. It appears as though two different processes have occurred. From the middle of the scratch a turning is removed, which is almost perfectly smooth (first process); at the edge of the scratch, however, that is, at the border line between turning and free glass surface, fine glass chips have been torn out (second process).

Due to an asymmetry of the scratching process, or of the scratching cube corner itself, chips are only deposited at one side of the scratch; the chips torn out from the other side can be seen sticking to one side of the turning, the other side of this turning being practically free from fine chips.

The turnings stand up from the glass surface a little, and in order to bring them sharply into focus the scratches themselves and the chips are somewhat diffuse, as is one of the turnings. The length of the longest turning is about $0.3 \mathrm{~mm}$. and the length of the scratches is $0.7 \mathrm{~mm}$.

These results appeared during the course of preliminary investigations of possible methods of distinguishing between diamonds of different hardness.

With respect to the formation of turnings and the plastic behaviour of glass under certain conditions, reference should also be made to research carried out by Ryschkewitsch ${ }^{2}$.

Diamond Research Laboratory, J. F. H. Custers

Industrial Distributors (1946), Ltd. 44 Main Street, Johannesburg.

${ }^{1}$ Taylor, E. W., Nature, 163, 323 (1949).

${ }^{2}$ Ryschkewitsch, E., Indust. Diam. Rev., 6, 339 (1946), 